Check for updates

Cite this: RSC Adv., 2017, 7, 43905

Received 14th July 2017

Accepted 6th September 2017

DOI: 10.1039/c7ra07743b

rsc.li/rsc-advances

\section{Solid-contact $\mathrm{Ca}^{2+}$-selective electrodes based on two-dimensional black phosphorus as ion-to- electron transducers $\uparrow$}

\author{
Lijuan Kou, ${ }^{\star a b}$ Minglang $\mathrm{Fu}^{\mathrm{a}}$ and Rongning Liang (D) *b
}

A new type of solid-contact polymeric membrane $\mathrm{Ca}^{2+}$-selective electrode has been developed by using black phosphorus as the transducing layer for the first time. The ion-to-electron transducing ability of black phosphorus was examined. The proposed electrode exhibits a good Nernstian slope with a detection limit of $4.0 \times 10^{-7} \mathrm{M}$.
Ion-selective electrodes (ISEs) have become a routine analytical tool in clinical diagnostics, process control, environmental and agricultural relevance. ${ }^{1-5}$ With a view to miniaturization, mass fabrication and measurements in small volumes, solid-contact ion-selective electrodes (SC-ISEs) with a solid inner contact as the ion-to-electron transducer, have been receiving much attention..$^{6-8}$

The first and most common SC-ISEs were constructed using polypyrrole (PPy), poly(3-octhylthiophene) (POT) and poly(3,4ethylene-dioxythiophene) (PEDOT) as the ion-to-electron transducers. ${ }^{9-11}$ However, these sensors may still suffer from problems of sensitivity to light and gases, undesired side reactions with the solid contact and the water layers. ${ }^{12}$ It is therefore highly desired to develop alternative materials as solid contacts for SC-ISEs. In recent years, SC-ISEs have subsequently experienced rapid growth which was mainly based on the development of nanomaterials. A wide variety of approaches that used carbon-based nanomaterials such as graphene and carbon nanotubes, gold nanoclusters and $\mathrm{MoS}_{2}$ nanoparticles as the ion-to-electron transducers have been successfully explored. ${ }^{13-16}$ Due to their large double layer capacitances, remarkable charge-transfer capability and high hydrophobicity, these SC-ISEs possess high potential stability and excellent resistance to gases and light. Therefore, the superior performance of these materials has led to the exploration of alternative two-dimensional materials as the ion-toelectron transducers for the development of SC-ISEs. ${ }^{17}$

${ }^{a}$ School of Enology, Binzhou Medical University, Yantai, Shandong 264003, P. R. China. E-mail: koulijuan@bzmc.edu.cn

${ }^{b}$ Key Laboratory of Coastal Environmental Processes and Ecological Remediation, Yantai Institute of Coastal Zone Research (YIC), Chinese Academy of Sciences (CAS), Shandong Provincial Key Laboratory of Coastal Zone Environmental Processes, YICCAS, Yantai, Shandong 264003, P. R. China. E-mail: rnliang@yic.ac.cn

$\dagger$ Electronic supplementary information (ESI) available. See DOI: $10.1039 / \mathrm{c} 7 \mathrm{ra} 07743 \mathrm{~b}$
Black phosphorus (BP), a new member of two-dimensional (2D) materials, has recently attracted global interest for both its fundamental properties and its applications. ${ }^{18-21}$ Recently, it has been reported that black phosphorus displays high charge carrier mobility and electrical conductance. ${ }^{19,22}$ These properties, together with its better surface-to-volume ratio, make BP a promising candidate for chemical sensing applications. Several papers have appeared recently including field-effect transistors for nitrogen dioxide $\left(\mathrm{NO}_{2}\right)$, electrochemical impedance spectroscopy for methanol and electrochemical sensing for humidity. ${ }^{23-25}$ However, without protection, black phosphorus flakes are reported to chemically degrade shortly with degradation of their electric properties and device performances. ${ }^{26} \mathrm{~A}$ recent theoretical paper demonstrated that black phosphorus flakes show improved stability for electrochemical sensing after being encapsulated by a hydrophobic polymeric membrane. ${ }^{27}$ Inspired by this, black phosphorus will be a very promising material for fabrication of SC-ISEs. As such, to the best of our knowledge, little is known about the performance of the black phosphorus as the ion-to-electron transducer of SCISEs.

Herein, we report here the first presenting of using black phosphorus for preparing the SC-ISEs. The $\mathrm{Ca}^{2+}$-selective electrode was chosen as a model system. The main performance characteristics of black phosphorus based SCISEs were estimated. The fabricated sensors exhibit excellent Nernstian responses and potential stability. No water layer or interferences by light, $\mathrm{O}_{2}$, or $\mathrm{CO}_{2}$ are observed.

The conducting film of SC-ISEs was prepared by drop-casting black phosphorus ethanol solution on electrode surface (see details in the ESI†). The morphology of black phosphorus powder on the electrode was characterized with a scanning electron microscope (SEM) on a ZEISS EVO LS15 instrument at an accelerating voltage of $10 \mathrm{kV}$. A panoramic picture of the black phosphorus conducting polymer is illustrated in Fig. 1. It can be seen that obtained black phosphorus dispersion 


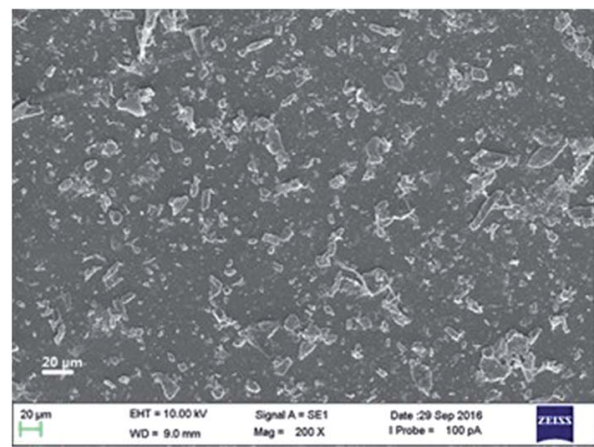

Fig. 1 SEM images of black phosphorus layers.

material shows high dispersity from each other. In addition, black phosphorus has an irregular shape with an average size of approximately $10 \mu \mathrm{m}$. When used in a SC-ISE, these materials are bound together by the poly(vinyl chloride) (PVC) binder as well as the plasticized polymeric membrane.

To further characterize the quality of black phosphorusbased solid contact, electrochemical impedance spectroscopy (EIS) was performed. EIS was performed with a three-electrode setup (see details in the ESI $\dagger$ ), where the glassy carbon electrode (GCE) coated with or without black phosphorus was connected as the working electrode, while an Au electrode as the auxiliary electrode and the $\mathrm{Ag} / \mathrm{AgCl}$ electrode as the reference electrode. Impedance measurements of GCE/black phosphorus/ $\mathrm{Ca}^{2+}$-ISE and GCE/Ca $\mathrm{Ca}^{2+}$-ISE recorded in $10^{-3} \mathrm{M} \mathrm{CaCl}_{2}$ are shown in Fig. 2. The diameter of the high-frequency semicircle is related to the bulk membrane coupled to the contact resistance between GC or solid contact and the ion-selective PVC membrane. The resistance of the GC/black phosphorus/ $\mathrm{Ca}^{2+}$ ISE $(0.64 \mathrm{M} \Omega)$ is smaller than that of the $\mathrm{GC} / \mathrm{Ca}^{2+}$-ISE $(1.13 \mathrm{M} \Omega)$, which indicates that the charge transfer across the interfaces is facilitated due to the presence of black phosphorus.

Current-reversal chronopotentiometry was used to evaluate the short-term potential stability of the black phosphorus-based $\mathrm{Ca}^{2+}$-ISE by recording potentials of the ISE under currents of \pm 1 $\mathrm{nA}$ in $1 \mathrm{mM} \mathrm{CaCl}_{2}$ solution. Fig. 3 shows that the potential drift of the $\mathrm{GC} / \mathrm{black}$ phosphorus $/ \mathrm{Ca}^{2+}$-ISE, derived from the ratio

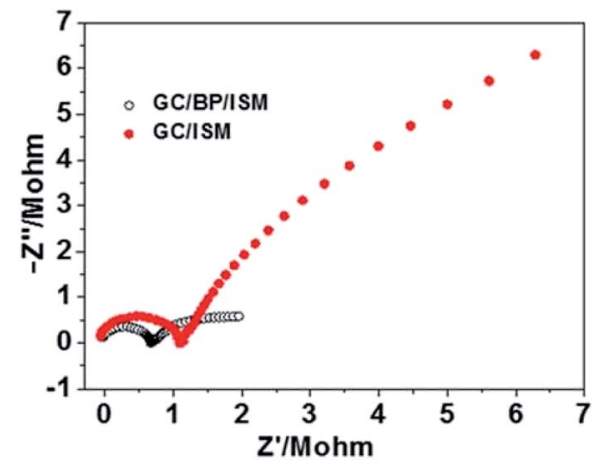

Fig. 2 Impedance spectra for the GC/black phosphorus/Ca ${ }^{2+}-$ ISE (hollow circle) and $\mathrm{GC} / \mathrm{Ca}^{2+}$-ISE (solid circle) in $10^{-3} \mathrm{M} \mathrm{CaCl}_{2}$. Frequency range, $0.3 \mathrm{~Hz}$ to $10 \mathrm{kHz} ; E_{\mathrm{dc}}, 0.2 \mathrm{~V} ; \Delta E_{\mathrm{dc}}, 10 \mathrm{mV}$.

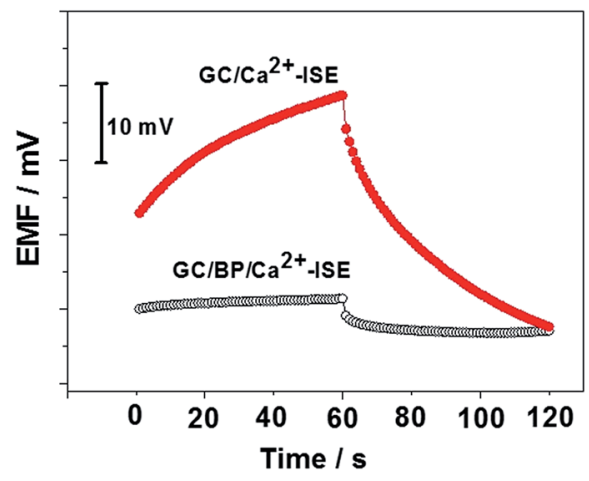

Fig. 3 Chronopotentiograms for the $\mathrm{GC} /$ black phosphorus/ $\mathrm{Ca}^{2+}-\mathrm{ISE}$ and $\mathrm{GC} / \mathrm{Ca}^{2+}$-ISE recorded in $10^{-3} \mathrm{M} \mathrm{CaCl}_{2}$.

$\Delta E / \Delta t$, is $72 \mu \mathrm{V} \mathrm{s}^{-1}$, which is much lower than that of the $\mathrm{GC} / \mathrm{Ca}^{2+}$-ISE under the same conditions $\left(500 \mu \mathrm{V} \mathrm{s}^{-1}\right)$. These results indicate that the potential stability of the electrode can be obviously improved by using the black phosphorus film as the ion-to-electron transducer.

The potentiometric performance characteristics such as the working concentration range, the limit of detection, the slope of the linear curve and response time were determined. The results of these measurements are summarized in Fig. 4. As can be seen, a linear range of the developed GC-BP- $\mathrm{Ca}^{2+}$-ISE from $1.0 \times 10^{-1}$ to $1.0 \times 10^{-6} \mathrm{M}$ is observed with a Nernst slope of $28.3 \pm 0.7 \mathrm{mV}$ decade $^{-1}\left(R^{2}=0.99\right)$. The detection limit calculated as the intersection of the slope line is $4.0 \times 10^{-7} \mathrm{M}$. The response time of the electrode was measured after successive immersion of the electrode in a series of $\mathrm{Ca}^{2+}$ solutions. A response time of about $10 \mathrm{~s}$ was required to achieve a steady potential within $0.7 \mathrm{mV}$ for measuring $\mathrm{Ca}^{2+}$ at concentrations ranging from $1.0 \times 10^{-1}$ to $1.0 \times 10^{-6} \mathrm{M}$. In addition, experiments also show that the potential response of the proposed electrode can be fully reversible with a relative standard deviation of $3.8 \%(n=5)$. The comparison of the analytical

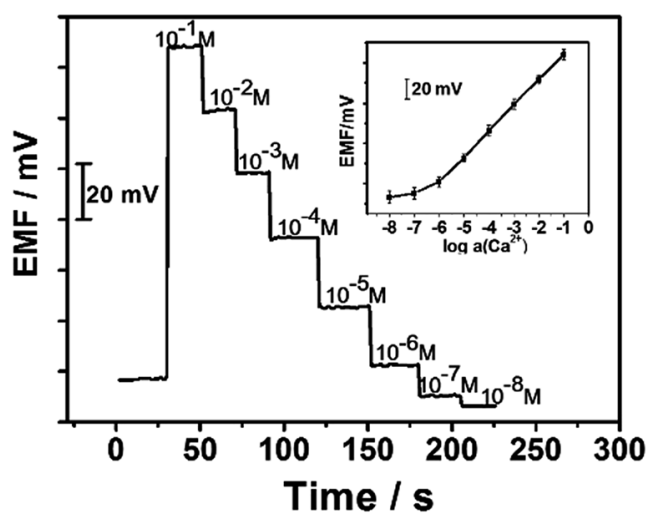

Fig. 4 Time trace of the GC/black phosphorus/ $\mathrm{Ca}^{2+}-\mathrm{ISE}$ in $\mathrm{CaCl}_{2}$ solution in the concentration range of $1.0 \times 10^{-8}$ to $1.0 \times 10^{-1} \mathrm{M}$. Inset shows the calibration curve of the GC/black phosphorus/Ca ${ }^{2+}-$ ISE. Each error bar represents one standard deviation for three measurements using the same electrode. 
performance of the developed sensor with some of other reported solid-contact ISEs is summarized in Table S1 in the ESI. $\dagger$ As illustrated, the response characteristics of the proposed electrode are comparable with those of other solid-contact ISEs.

The potentiometric selectivity coefficients were obtained in an alkali metal or alkaline earth metal chloride solutions using the separate solution method. The results are presented in Table S1. $\dagger$ As can be seen, GC-black phosphorus-Ca ${ }^{2+}$-ISEs exhibited a high selectivity over interfering ions, which are all very close to the values reported in the literature. These phenomena indicate that the selectivity of the developed $\mathrm{Ca}^{2+}$ ISEs is not affected by the ion-to-electron transducing layer, but is dependent on the ionic sensing polymeric membrane itself. This selectivity satisfied the requirements for calcium assay in the wine samples.

A potentiometric water layer test was conducted with all electrodes to evaluate the water layer formation between the polymeric ion-selective membrane and its solid contact. The electrode was first immersed in $0.1 \mathrm{~mol} \mathrm{~L}^{-1}$ of $\mathrm{CaCl}_{2}$ solution. Then the solution was changed to $0.1 \mathrm{~mol} \mathrm{~L}^{-1} \mathrm{NaCl}$ solution. After $2 \mathrm{~h}$ the $\mathrm{NaCl}$ solution was replaced by $0.1 \mathrm{~mol} \mathrm{~L}^{-1}$ of $\mathrm{CaCl}_{2}$ solution. The potential responses of the tested electrodes are shown in Fig. 5. As can be seen, a negative EMF change is observed for both electrodes upon replacing the $\mathrm{CaCl}_{2}$ solution with the $\mathrm{NaCl}$ solution. Such EMF change reflects the change in the outer phase boundary potential as a consequence of the selectivity behavior of the $\mathrm{Ca}^{2+}$-selective membrane. In this process, the electrode based on black phosphorus exhibits a stable potential response while an obvious positive potential drift is observed for the electrode without solid contact. These results demonstrate that no undesirable water layer is formed for the GC/black phosphorus-Ca ${ }^{2+}$-ISE while there was a water layer at the sensing polymer/electrode interface in case of absence of the ion-to-electron transducer layer.

It has been reported that light, oxygen, and carbon dioxide may cause interference to several SC-ISEs. Oxygen or carbon dioxide can permeate plasticized PVC membranes which affect the boundary potential or alter the local $\mathrm{pH}$. In addition, SC-ISE can be photosensitive if the solid contact is an organic semiconductor. In this study, the effect of $\mathrm{O}_{2}$ and $\mathrm{CO}_{2}$ was tested by bubbling both gases into $10^{-3} \mathrm{M} \mathrm{CaCl}_{2}$ solution for $25 \mathrm{~min}$

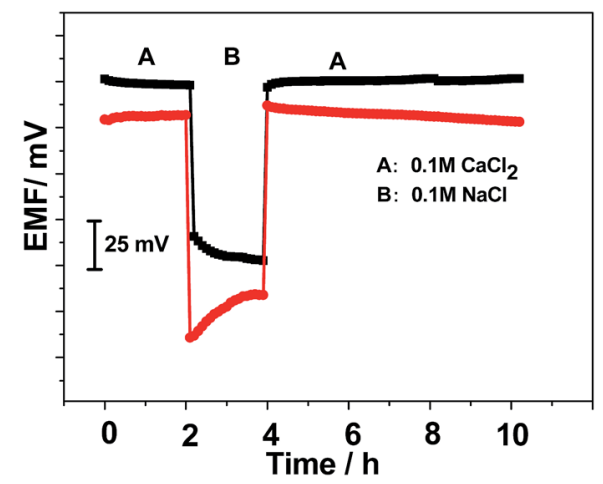

Fig. 5 Water layer tests for the GC/black phosphorus/Ca ${ }^{2+}-$ ISE and $\mathrm{GC} / \mathrm{Ca}^{2+}-\mathrm{ISE}$ in $0.1 \mathrm{M} \mathrm{CaCl}_{2}(\mathrm{~A})$ and $0.1 \mathrm{M} \mathrm{NaCl}(\mathrm{B})$.

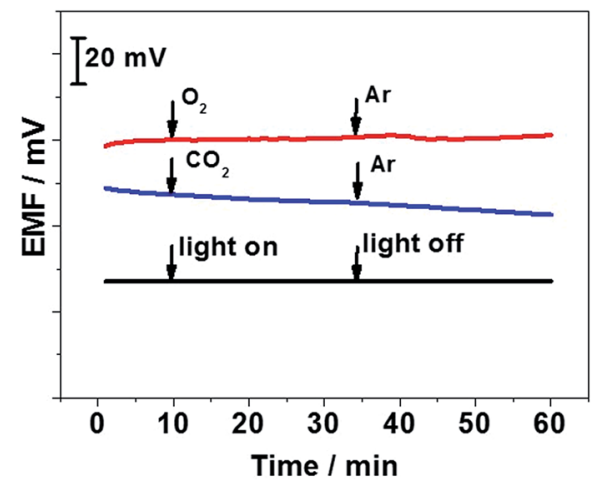

Fig. 6 Effects of light, $\mathrm{O}_{2}$, and $\mathrm{CO}_{2}$ on the potential stability of the $\mathrm{GC}$ / black phosphorus/ $\mathrm{Ca}^{2+}-$ ISE in $10^{-3} \mathrm{M} \mathrm{CaCl}_{2}$ solution.

respectively followed by purging with $\mathrm{N}_{2}$ to remove $\mathrm{O}_{2}$ or $\mathrm{CO}_{2}$ for $25 \mathrm{~min}$. In addition, the effect of light on the GC/BP-Ca ${ }^{2+}$-ISE was investigated by recording the potential response in a $10^{-3} \mathrm{M} \mathrm{CaCl}_{2}$ solution while turning on/off the ambient light. As clearly illustrated in Fig. 6, no significant potential changes are observed during the measurements, indicating that the black phosphorus film-based solid contact has no light, $\mathrm{O}_{2}$ or $\mathrm{CO}_{2}$ sensitivity.

The proposed GC/black phosphorus $/ \mathrm{Ca}^{2+}$-ISE was finally applied to determination of $\mathrm{Ca}^{2+}$ in wine samples that are widely consumed by the local public. It should be noted that alcohol was removed from wine samples using rotary evaporators and the samples were diluted 10 times with deionized water before potentiometric detection. In order to illustrate its accuracy, the comparison between the proposed sensor and the atomic absorption spectrophotometry (AAS) method was performed. ${ }^{28}$ The results are given in Table S2. $\dagger$ It can be seen the data obtained by the proposed electrode agree well with those obtained by the AAS method, indicating that the proposed GC/ black phosphorus/ $\mathrm{Ca}^{2+}$-ISE has a promising potential for real sample analysis.

\section{Conclusions}

In summary, we have for the first time used a few-layer black phosphorus film as the ion-to-electron transducer in solidcontact $\mathrm{Ca}^{2+}$-ISE. The presence of this layer significantly reduces the resistance of membrane compared to coated disk electrode. There are also many other important advantages, including long-term potential stability, no undesirable water layer and their insensitivity to $\mathrm{O}_{2}$ or $\mathrm{CO}_{2}$ and light. In addition, the electrodes demonstrate excellent sensing properties including fast response time, a wide dynamic response range, low detection limit and good selectivity. Moreover, this robust sensor was successfully applied for $\mathrm{Ca}^{2+}$ determination in the wine samples with similar results to AAS.

\section{Conflicts of interest}

There are no conflicts to declare. 


\section{Acknowledgements}

This work was financially supported by the Natural Science Foundation of Shandong province (BS2014NY004), the National Natural Science Foundation of China (41576106), the Science and Technology Project of Yantai (2014ZH086), the Youth Innovation Promotion Association of CAS (2014190). Many thanks to Prof. Wei Qin's support for the whole experiment.

\section{Notes and references}

1 E. Bakker, Anal. Chem., 2016, 88, 395-413.

2 T. Guinovart, D. Hernandez-Alonso, L. Adriaenssens, P. Blondeau, F. X. Rius, P. Ballester and F. J. Andrade, Biosens. Bioelectron., 2017, 87, 587-592.

3 V. K. Gupta, M. R. Ganjali, P. Norouzi, H. Khani, A. Nayak and S. Agarwal, Crit. Rev. Anal. Chem., 2011, 41, 282-313.

4 C. Z. Lai, M. A. Fierke, R. C. da Costa, J. A. Gladysz, A. Stein and P. Bühlmann, Anal. Chem., 2010, 82, 7634-7640.

5 S. T. Mensah, Y. Gonzalez, P. Calvo-Marzal and K. Y. Chumbimuni-Torres, Anal. Chem., 2014, 86, 7269-7273.

6 G. A. Crespo, S. Macho and F. X. Rius, Anal. Chem., 2008, 80, 1316-1322.

7 M. A. Fierke, C. Z. Lai, P. Bühlmann and A. Stein, Anal. Chem., 2010, 82, 680-688.

8 J. P. Veder, R. De Marco, G. Clarke, R. Chester, A. Nelson, K. Prince, E. Pretsch and E. Bakker, Anal. Chem., 2008, 80, 6731-6740.

9 J. Sutter and E. Pretsch, Electroanalysis, 2006, 18, 19-25.

10 P. Sjoberg-Eerola, J. Nylund, J. Bobacka, A. Lewenstam and A. Ivaska, Sens. Actuators, B, 2008, 134, 878-886.

11 M. Wagner, G. Lisak, A. Ivaska and J. Bobacka, Sens. Actuators, B, 2013, 181, 694-701.

12 J. Sutter, E. Lindner, R. E. Gyurcsányi and E. Pretsch, Anal. Bioanal. Chem., 2004, 380, 7-14.

13 G. A. Crespo, S. Macho and F. X. Rius, Anal. Chem., 2008, 80, 1316-1322.
14 Z. A. Boeva and T. Lindfors, Sens. Actuators, B, 2016, 224, 624-631.

15 J. A. Xu, F. Jia, F. H. Li, Q. B. An, S. Y. Gan, Q. X. Zhang, A. Ivaska and L. Niu, Electrochim. Acta, 2016, 222, 1007-1012.

16 X. Z. Zeng, S. Y. Yu, Q. Yuan and W. Qin, Sens. Actuators, B, 2016, 234, 80-83.

17 C. Bieg, K. Fuchsberger and M. Stelzle, Anal. Bioanal. Chem., 2017, 409, 45-61.

18 A. Castellanos-Gomez, J. Phys. Chem. Lett., 2015, 6, 42804291.

19 C. C. Mayorga-Martinez, Z. Sofer and M. Pumera, Angew. Chem., Int. Ed., 2015, 54, 14317-14320.

20 H. U. Lee, S. Y. Park, S. C. Lee, S. Choi, S. Seo, H. Kim, J. Won, K. Choi, K. S. Kang, H. G. Park, H.-S. Kim, H. R. An, K.-H. Jeong, Y.-C. Lee and J. Lee, Small, 2016, 12, 214-219.

21 Z. Sofer, D. Bousa, J. Luxa, V. Mazanek and M. Pumera, Chem. Commun., 2016, 52, 1563-1566.

22 Z. Sofer, D. Sedmidubsky, S. Huber, J. Luxa, D. Bousa, C. Boothroyd and M. Pumera, Angew. Chem., Int. Ed., 2016, 55, 3382-3386.

23 A. N. Abbas, B. Liu, L. Chen, Y. Ma, S. Cong, N. Aroonyadet, M. Koepf, T. Nilges and C. Zhou, ACS Nano, 2015, 9, 56185624.

24 P. Yasaei, A. Behranginia, T. Foroozan, M. Asadi, K. Kim, F. Khalili-Araghi and A. Salehi-Khojin, ACS Nano, 2015, 9, 9898-9905.

25 Y. Du, H. Liu, Y. Deng and P. D. Ye, ACS Nano, 2014, 8, 10035-10042.

26 S. Gamage, Z. Li, V. S. Yakovlev, C. Lewis, H. Wang, S. B. Cronin and Y. Abate, Adv. Mater. Interfaces, 2016, 3, 1600121.

27 P. Li, D. Z. Zhang, J. J. Liu, H. Y. Chang, Y. Sun and N. L. Yin, ACS Appl. Mater. Interfaces, 2015, 7, 24396-24402.

28 R. Sowmya, K. P. Indumathi, S. Arora, V. Sharma and A. K. Singh, J. Food Sci. Technol., 2015, 52, 1188-1193. 